\title{
Diet and physical activity as determinants of weight gain, overweight and obesity: The WCRF/AICR evidence and policy implications
}

\author{
$\underline{\text { Isobel Bandurek }}^{1}$, Emily Almond ${ }^{1}$, Susannah Brown ${ }^{1}$, Giota Mitrou ${ }^{1}$, Ifigeneia Bourgiezi ${ }^{1}$, \\ Nigel Brockton ${ }^{2}$, Deirdre McGinley-Gieser ${ }^{2}$, Martin Wiseman ${ }^{1}$, Fiona Sing ${ }^{1}$, \\ Michael Leitzmann ${ }^{3}$ and Kate Allen ${ }^{1}$ \\ ${ }^{1}$ WCRF International, London, United Kingdom, \\ ${ }^{2}$ American Institute for Cancer Research, Arlington, VA, USA and \\ ${ }^{3}$ University of Regensburg, Regensburg, Germany
}

\section{Abstract}

Introduction: Globally, over 1.97 billion adults and 338 million children and adolescents are living with overweight and obesity, increasing the risk of numerous co-morbidities, including at least 12 cancers ${ }^{(1)}$. WCRF/AICR conducted a literature review of diet and physical activity as determinants of weight gain, overweight and obesity in adults and children. We also introduce a novel evidence-based policy framework for promoting physical activity, and linked database, currently in development as part of the EU-funded CO-CREATE project on child and adolescent obesity prevention.

Materials and Methods: Evidence on diet and physical activity as determinants and risk of weight gain, overweight and obesity was systematically extracted from existing reviews and a systematic search for recent meta-analyses, then collated and analysed. The WCRF Continuous Update Project Expert Panel drew conclusions about which exposures influence risk of weight gain, overweight and obesity, using pre-defined criteria that included evidence of biological plausibility.

Results: The Panel identified strong evidence that several diet and physical activity related exposures influence the risk of weight gain, overweight and obesity in adults and children (see table 1). Separate conclusions were drawn for adults and children in relation to screen time, considered a marker of sedentary time.

However, the Panel noted that as exposures tend to cluster, physiologically interact and share common biological mechanisms, they should not be regarded as absolutely 'singular'but an integrated concept of interrelated exposures within a pattern of lifestyle.

For full list of footnotes, see Energy Balance and Body Fatness report ${ }^{(1)}$.

Discussion: Healthy dietary patterns help prevent excess weight gain. Achieving such patterns requires attention to the broader economic, environmental and social factors that influence and constrain people's behaviour. The findings of this report support the need for evidence-based public health policy to help create health-enabling environments, particularly for children and adolescents. The WCRF International MOVING framework ${ }^{(2)}$ presents a package of policies to promote physical activity, which alongside wider public health policy can help address the multiple drivers of overweight and obesity.

Table 1. Risk of weight gain, overweight and obesity

\begin{tabular}{lll}
\hline STRONG EVIDENCE & DECREASES RISK & INCREASES RISK \\
\hline CONVINCING & Walking & $\begin{array}{l}\text { Screen time (children) } \\
\text { Sugar sweetened drinks }\end{array}$ \\
PROBABLE & Aerobic physical activity & $\begin{array}{l}\text { Screen time (adults) } \\
\text { 'Fast foods' }\end{array}$ \\
& $\begin{array}{l}\text { Foods containing dietary fibre } \\
\text { 'Mediterranean type' dietary pattern } \\
\text { Having been breastfed }\end{array}$ & \\
\hline
\end{tabular}

\section{Acknowledgements}

WCRF/AICR Continuous Update Project Panel, chaired by Professor Alan Jackson CUP Team at Imperial College London, led by Dr Teresa Norat and Dr Doris Chan.

\section{Conflict of Interest}

There is no conflict of interest.

\section{References}

1. WCRF/AICR. (2018) Diet, Nutrition, Physical Activity and Cancer: A Global Perspective. CUP Expert Report. Available at dietandcancerreport. org

2. WCRF International (2019) MOVING framework. Available at wcrf.org 